\title{
Assembly of Oriented Ultrafine Polymer Fibers by Centrifugal Electrospinning
}

\author{
Shu-Liang Liu, ${ }^{1}$ Yun-Ze Long, ${ }^{1,2,3}$ Zhi-Hua Zhang, ${ }^{1}$ Hong-Di Zhang, ${ }^{1,2}$ \\ Bin Sun, ${ }^{1,2,4}$ Jun-Cheng Zhang, ${ }^{1,2}$ and Wen-Peng Han ${ }^{1,2}$ \\ ${ }^{1}$ College of Physics, Qingdao University, Qingdao 266071, China \\ ${ }^{2}$ Key Laboratory of Photonics Materials and Technology in Universities of Shandong (Qingdao University), Qingdao 266071, China \\ ${ }^{3}$ State Key Laboratory Cultivation Base of New Fiber Materials and Modern Textile, Qingdao University, Qingdao 266071, China \\ ${ }^{4}$ College of Chemistry, Chemical Engineering \& Environment, Qingdao University, Qingdao 266071, China
}

Correspondence should be addressed to Yun-Ze Long; yunze.long@163.com

Received 29 May 2013; Accepted 9 October 2013

Academic Editor: Raymond Whitby

Copyright (C) 2013 Shu-Liang Liu et al. This is an open access article distributed under the Creative Commons Attribution License, which permits unrestricted use, distribution, and reproduction in any medium, provided the original work is properly cited.

\begin{abstract}
Uniaxially aligned and cross-aligned arrays of ultrafine polymer fibers have been fabricated by a novel and effective centrifugal electrospinning setup with rotating polymer solution jets. Comparing with conventional electrospinning (10-30 kV) and centrifugal spinning $(4,000-12,000 \mathrm{rpm})$, this technique only requires a lower working voltage $(2.8-6.0 \mathrm{kV})$, a slower rotational speed (360$540 \mathrm{rpm})$, and a shorter spinning distance $(2.0-4.0 \mathrm{~cm})$. In addition, the influences of experimental parameters such as working voltage, rotational speed, collecting distance, and solution concentration on the alignment of the as-spun fibers are investigated using image analysis techniques. It is found that the working voltage and rotational speed mainly influence the perpendicular and linear velocities of the fibers, respectively. The polymer fibers tend to show higher alignment degree when these two velocities are very close. Optimum conditions (working voltage $3.0 \mathrm{kV}$, rotational speed $420 \mathrm{rpm}$, collecting distance $2.5 \mathrm{~cm}$, and solution concentration $18 \mathrm{wt} \%)$ to maximize alignment degree ( 97\%) of polystyrene fibers are also obtained.
\end{abstract}

\section{Introduction}

One-dimensional (1D) ultrafine structures have been extensively reported in recent years as potential building blocks for flexible electronics, (bio)chemical sensors, optoelectronics, energy harvesting and storage materials $[1-5]$. For practical applications, it is necessary to large-scale assemble 1D nanostructures on various rigid or flexible substrates. By now, a variety of techniques, namely, assembly by pregrown nanowires and assembly by direct growth, have been developed to achieve controlled and uniform assembly of nanowires, including flow-assisted alignment, Langmuir-Blodgett assembly, bubble-blown technique, electric- or magneticfield-directed assembly, contact or roll printing, bridging method, and electrospinning [5].

Comparing with other fabrication and assembly methods, electrospinning is an efficient and highly versatile technique which can be further developed for mass production of uniform, ultrafine, and continuous fibers [6]. Ultrafine fibers fabricated by conventional electrospinning are usually without orientation as a piece of nonwoven film, which may limit the potential applications of electrospun fibers, especially in the fields of (opto)electronics, photonics and actuators which need direct, fast charge transfer, or regular, uniform structures [5]. In the last decade, various modified electrospinning techniques have been proposed or developed to fabricate aligned fibers, including parallel pair electrodes collection [79], rotating drum or disc collection $[10,11]$, frame collector [6, $12]$, step collector [13], auxiliary electric $[14,15]$ or magnetic $[16,17]$ electrospinning, centrifugal electrospinning [1821], double spinning [22], and electroconductive template collection [23]. Although the orientation of electrospun fibers may be controlled to a certain extent by these methods, some drawbacks still exist and should be removed for practical application. For example, in the parallel pair electrodes method, the aligned fibers are restricted by narrow area and 
limited length. In the rotating drum method, the degree of fiber alignment is not so acceptable and decreases with the increase of spinning time.

Among these methods, centrifugal electrospinning, a new method reported in recent years, combines the advantages of centrifugal spinning [24-26] and conventional electrospinning effectively. Namely, centrifugal electrospinning combines the centrifugal force and electrical force into the process of spinning, and thus high alignment of the as-spun fibers and higher production rate can be achieved at a lower working voltage or slower rotating velocity. For instance, several papers [18-21] have reported rotating method to generate aligned electrospun fibers. In their electrospinning apparatus, the rotating polymer solution container and nozzle are located at the center of the collector, a hollow metallic cylinder. The cylinder is not moving and the spun fibers will pull towards it. Due to opposite charges between the nozzle and the cylinder, the emerging jet from the nozzle tip rotates onto the interior surface of the cylinder. It is found that the alignment degree of electrospun fibers can reach $70.2 \%$, but the working voltage $(10-22 \mathrm{kV}[20,21]$ and $25 \mathrm{kV}$ [19]) and rotational speed (1800 rpm [19] and 2385 and $6360 \mathrm{rpm}$ [20]) are still very high.

In this paper, a new configuration of centrifugal electrospinning setup is proposed to decrease both the centrifugal field and electrical field simultaneously. However, because of lower working voltage and rotational speed, diameters of as-spun fibers distribute in a wide range from several hundred nanometers to a few microns. So, fibers produced by centrifugal electrospinning could be termed as ultrafine fibers or microfibers. Beside highly aligned polymer fiber arrays, crossed fluorescent fiber grids are also successfully assembled by this setup. The results indicate that centrifugal electrospinning can be used in large-scale integration of aligned ultrafine fibers and devices.

\section{Experimental}

2.1. Materials. Polystyrene (PS, GR, Mw 250,000, Acros Organics) and polymethyl methacrylate (PMMA, GR, Mw 350,000, Sigma-Aldrich) were dissolved into tetrahydrofuran (THF), and polyvinylpyrrolidone (PVP, AR, Mw 1,300,000, Aldrich) was dissolved into ethanol to prepare precursor solutions. Fluorescein (AR, Mw $=332.31$, Shanghai Medical Chemicals Reagent), rhodamine B (AR, Mw $=479.01$, Heguang pure medical industry), and home-made $\mathrm{Eu}$ (aspirin) ${ }_{3}$ (Phen) [27] were used as dopant to improve the fluorescence properties of the electrospun fibers. All the materials were used as received without further treatment and all the experiments were performed at room temperature.

2.2. Centrifugal Electrospinning Setup. The used setup of centrifugal electrospinning is based on the conventional electrospinning and centrifugal spinning. As shown in Figure 1, a syringe with a right-angle needle is fixed horizontally on a circular platform termed "support disk," which is attached to a speed-adjustable motor through an insulated axis, and the rotating radius of the needle is $16 \mathrm{~cm}$ (distance of the needle tip to the shaft). The collector is a static annular aluminum foil

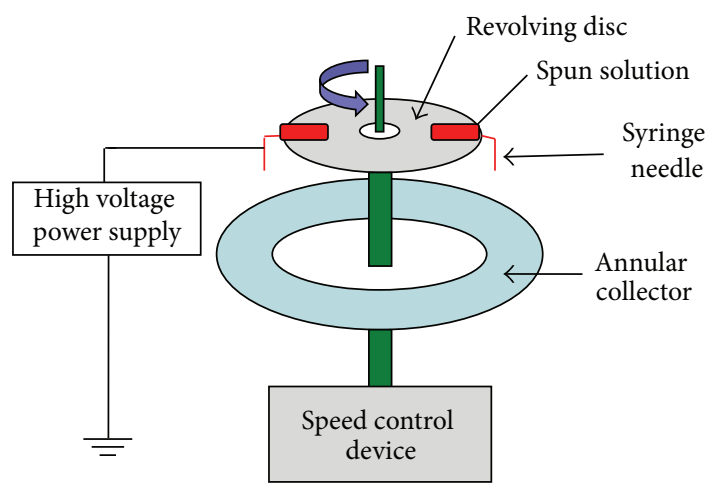

FIgURE 1: Schematic illustration of the centrifugal electrospinning apparatus.

(inner diameter $20 \mathrm{~cm}$ and outer diameter $44 \mathrm{~cm}$ ) and located under the support disk. High voltage is applied between the syringe needle and collector. Ultrafine fibers are collected on glass slice, which is placed on the static annular collector. The polymeric jet emanated from the top of Tailor Cone that located at the tip of syringe needle under the action of electrical force and centrifugal force. At first, the jet follows a path that starts with a straight segment and the segment moves towards the collector until the first bending instability develops, and then the jets are elongated by the repulsive force of charges on the jets [28]. Ultrafine fibers are fabricated after the jets solidify and fall onto the collector.

2.3. Characterizations. The microscale morphologies of fibers were characterized by a scanning electron microscope (SEM; JEOL JSM-6390). The optical images were obtained by an optical microscope (Motic SMZ-168). The fluorescence images of the as-spun fibers were taken by a Leica DM6000B fluorescent microscope.

\section{Result and Discussion}

To maximize fibers alignment, the influences of various experimental parameters such as working voltage, rotational speed, collecting distance, and concentration of polymer solution on morphology and alignment of the as-spun microfibers were investigated. And thus highly aligned polymer nanofibers were fabricated by the centrifugal electrospinning at the optimized processing variables.

3.1. Collecting Distance. In conventional electrospinning, the collecting distance is about $10.0-20.0 \mathrm{~cm}$, and the as-spun microfibers usually display as the form of nonwovenlones. According to Reneker's theory [28], the onset of bending instability inevitably makes the jets curve, and then after several turns are formed, a new electrical bending instability formed smaller coils on the previous larger coils. Many new smaller bending coils will emerge until the jets are solidified into nanofibers. When the collecting distance is within $5.0 \mathrm{~cm}$, the bending of the jets occurred prior to the impingement onto the surface of collector $[28,29]$. In our devise, centrifugal force provides a horizontal velocity for the coiled 


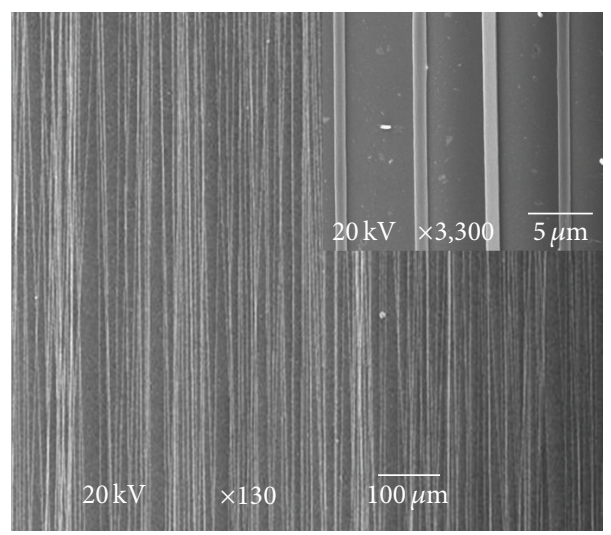

(a)

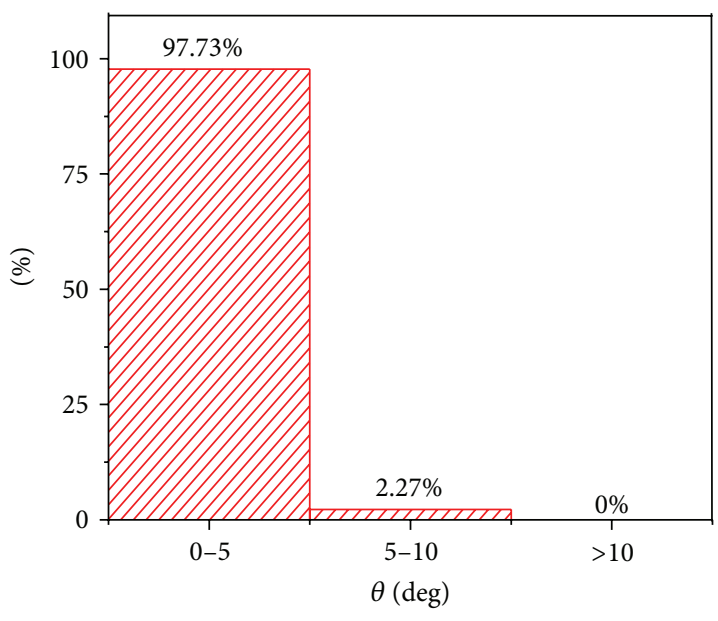

(b)

FIGURE 2: (a) SEM images of aligned straight PS fibers electrospun at conditions of $3.0 \mathrm{kV}, 420 \mathrm{rpm}$, and $2.5 \mathrm{~cm}$. Inserted is a SEM image with larger magnification. (b) Distributions of the degree of alignment $(\gamma)$ defined by the difference between the angles of orientation $(\theta)$ of fibers and the long axis.

jet, which draws the jet moving in horizontal direction, and thus the deposited fibers become straight and aligned. If all the fibers on the collector are considered to possess uniform morphology, the collecting distance should be controlled within the distance of the first bending coil. Since the straight segment of the jets is about $1.0 \mathrm{~cm} \mathrm{[29],} \mathrm{we} \mathrm{propose} \mathrm{that} \mathrm{the}$ collecting distance should be less than $5.0 \mathrm{~cm}$, for example, between 2.0 and $4.0 \mathrm{~cm}$. A shorter distance does not facilitate solvent evaporation and jet solidification. However, a larger distance means that a higher rotational speed is needed to pull the jets straight and aligned.

Figure 2 shows the SEM images of highly aligned straight polystyrene (PS) fibers (the concentration was $18 \mathrm{wt} \%$ ), which were fabricated at the optimized conditions (the collecting distance was $2.5 \mathrm{~cm}$ ). We found that more than $97.7 \%$ of the PS fibers are within $\theta=5^{\circ}$, and all of the rest are still within $\theta=$ $10^{\circ}$, as shown in Figure 2(b). If we define $\gamma$ as the percentage of fibers with $\theta<5^{\circ}$, our new centrifugal electrospinning setup can produce well-aligned fibers with $\gamma=97.7 \pm 2.0 \%$. This value of alignment degree is much higher than that produced by other centrifugal spinning [24-26] and centrifugal electrospinning [18-21] methods.

\subsection{Working Voltage and Rotational Velocity}

3.2.1. The Relationship between Applied Voltage and Rotation Velocity. The working voltage is one of the main parameters that influence the morphology of electrospun fibers [30]. The rotational velocity also influences the process where jets solidify into fibers [26]. At the beginning, the straight segment of the jet is mainly controlled by two forces: electrical force and centrifugal force. The motion of the straight segment in vertical direction can be depicted by (1). Herein, $U$ is the working voltage and $v$ is the velocity of the jet in the strong electrostatic field. In horizontal direction, microfibers move at the constant velocity generated by the revolving

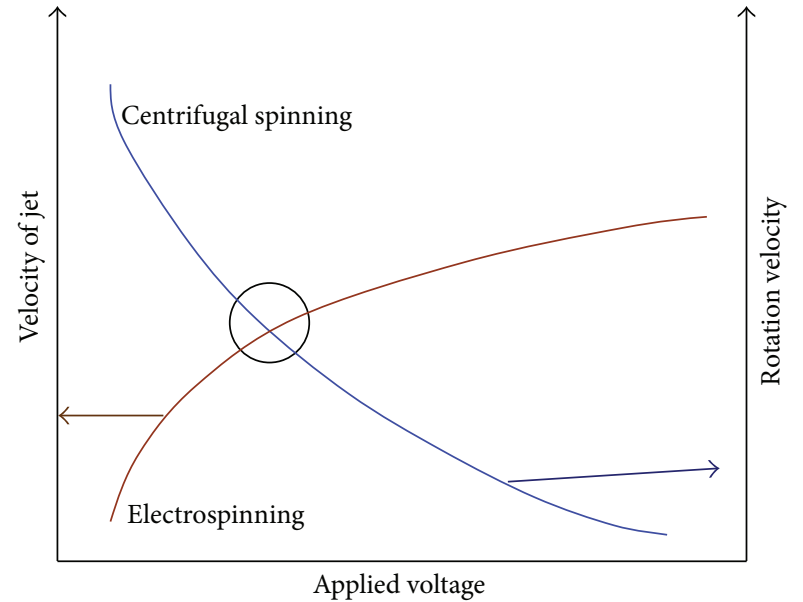

FIGURE 3: Schematic diagram of the relationship of applied voltage with velocity of polymer jet and rotational velocity.

needle, given by (2); here $\nu_{\perp}$ is the tangential velocity of the needle:

$$
\begin{gathered}
e U=\frac{1}{2} m v^{2}, \\
\nu_{\perp}=\omega r .
\end{gathered}
$$

Figure 3 describes the relationship between the velocity of jet and applied voltage qualitatively. In practical experiments, if the needle keeps static, the setup becomes conventional electrospinning; disordered fibers will be collected. With the increase of the rotational velocity, centrifugal force (rotational velocity) of the jet increases and partly replaces the electric force to overcome the surface tension, which leads to the decrease of applied voltage. And the horizontal motion of the needle can improve the fiber alignment. When the applied 




(a)



(b)

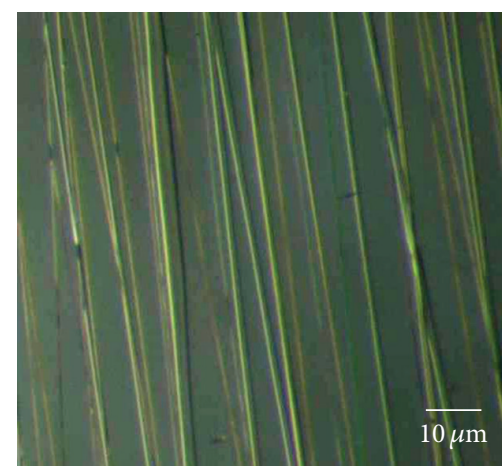

(c)

FIGURE 4: Optical microscope images of PVP fibers electrospun at conditions of $6.2 \mathrm{kV}$ and $3.0 \mathrm{~cm}$ with rotational velocity of (a) $360 \mathrm{rpm}$, (b) $420 \mathrm{rpm}$, and (c) $540 \mathrm{rpm}$.

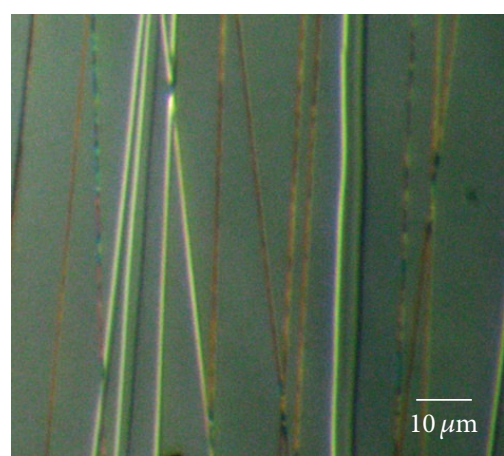

(a)

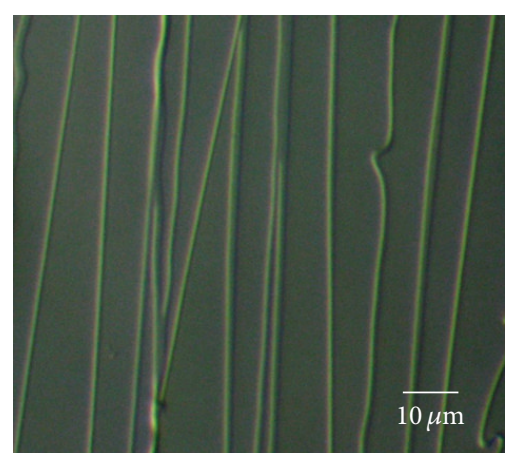

(b)

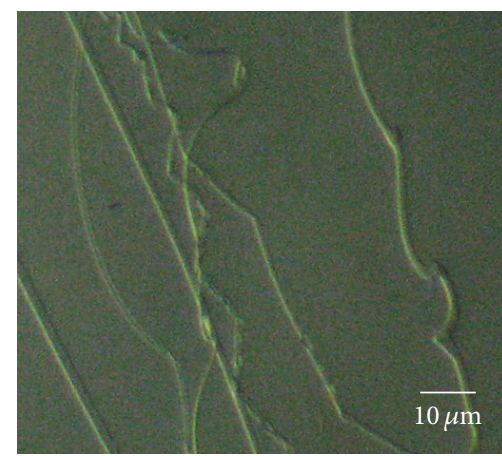

(c)

Figure 5: Optical microscope images of PVP fibers electrospun at conditions of $420 \mathrm{rpm}$ and $3.0 \mathrm{~cm}$ with applied voltage of (a) $4.0 \mathrm{kV}$, (b) $6.0 \mathrm{kV}$, and (c) $8.0 \mathrm{kV}$.

voltage decreases to $0 \mathrm{~V}$ and the rotational velocity increases to thousands of rpm, the setup becomes centrifugal spinning. And in this condition, it is difficult to produce highly aligned microfibers with uniform morphology [26]. In fact, it has been observed that polymer fibers tend to be aligned when the perpendicular and linear velocities are very close or at the same order of magnitude [31]. Thus, the optimum condition for aligned microfibers with fine morphology is limited in the black circle, as shown in Figure 3.

3.2.2. Rotational Velocity. When the syringe (needle-to-axis rotating radius is $16 \mathrm{~cm}$ ) has been fixed, different rotational velocity reflects different centrifugal force. For example, rotational velocity of centrifugal spinning is about thousands of rpm [26]. In centrifugal electrospinning, due to the electronic force partly taking the place of centrifugal force to get over the surface tension, the rotational velocity need not be very large. Experimental results indicate that the velocity from 300 to $600 \mathrm{rpm}$ is large enough to meet the request. Faster velocity or slower velocity cannot facilitate the alignment degree of fibers. In order to simplify the experiments, the velocitycontrolled device provides three velocities, which let the disc revolve at three different rotational velocities $(360,420$, and $540 \mathrm{rpm}$, resp.). Figure 4 shows the optical microscope images of PVP fibers with these three rotational velocities. They were all fabricated at the conditions that applied voltage was $6.2 \mathrm{kV}$ and collecting distance was $3.0 \mathrm{~cm}$. It can be seen that $420 \mathrm{rpm}$ is more suitable for the applied voltage $(6.2 \mathrm{kV})$, and at these conditions the polymer fibers fabricated are relatively parallel, as shown in Figure 4(b). Slower rotational velocity (360 rpm) cannot provide enough horizontal velocity to pull the jet fully straight (Figure 4(a)).

3.2.3. Working Voltage. The above results exhibit that the rotational velocity of $420 \mathrm{rpm}$ can produce highly aligned fiber arrays. So we adopt $420 \mathrm{rpm}$ to study the influence of working voltage. Figure 5 gives the optical microscope images of PVP fibers with different applied voltages. When the applied voltage was at $6.0 \mathrm{kV}$ (Figure 5(b)), we can achieve the most aligned fibers compared with 4.0 (Figure 5(a)) and $8.0 \mathrm{kV}$ (Figure 5(c)). At the same polymer concentration, the alignment degree of PVP fibers firstly ascended and then descended with the increase of the applied voltage. When the applied voltage is low (Figure 5(a)), PVP fibers show columnar structure. This is because low applied voltage offers small electrical force so that the diameter of the coiled jets cannot be elongated. The diameter of the jets is too thick to solidify into microfibers. On the contrary, when applied 


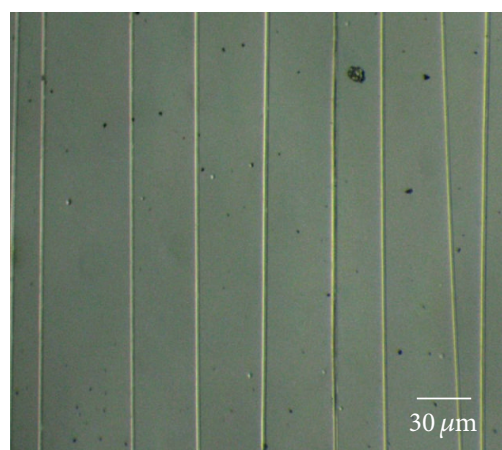

(a)

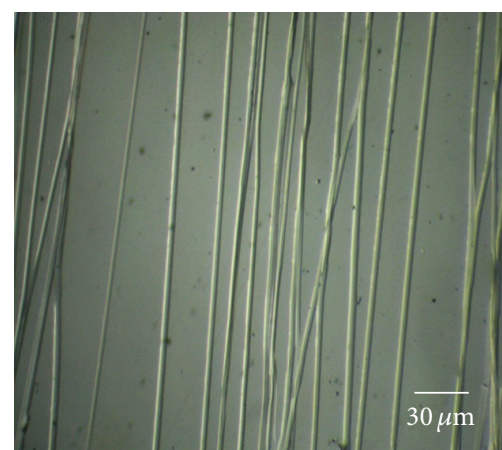

(b)

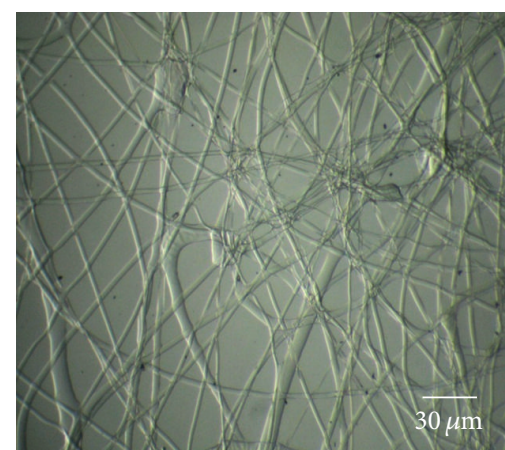

(c)

Figure 6: Optical microscope images of PMMA fibers electrospun at conditions of $420 \mathrm{rpm}$ and $2.0 \mathrm{~cm}$ with applied voltage of (a) $4.5 \mathrm{kV}$, (b) $6.0 \mathrm{kV}$, and (c) $7.0 \mathrm{kV}$.

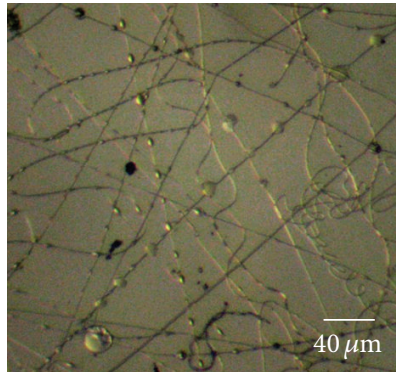

(a)

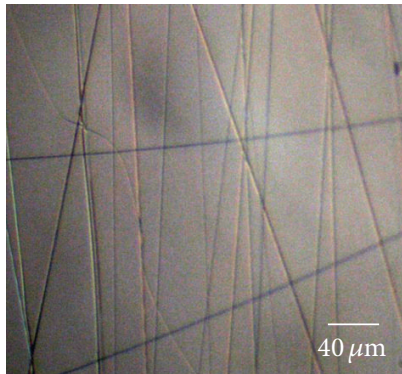

(b)

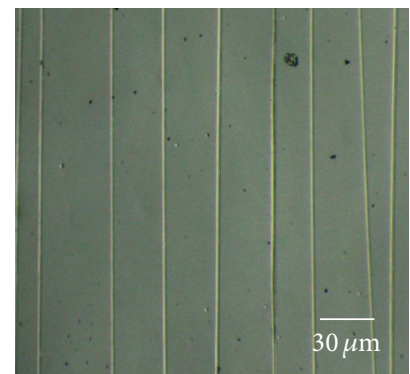

(c)

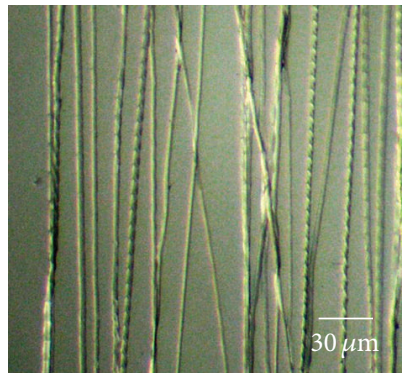

(d)

FIGURE 7: Optical microscope images of PMMA fibers electrospun at conditions of $4.5 \mathrm{kV}, 420 \mathrm{rpm}$ and $2.0 \mathrm{~cm}$ with PMMA/THF solution concentration of (a) $8.0 \mathrm{wt} \%$, (b) $10.0 \mathrm{wt} \%$, (c) $13.0 \mathrm{wt} \%$, and (d) $15.0 \mathrm{wt} \%$, respectively.

voltage is too high, coiled jets are overelongated and the diameters of the jets may decrease. At this time, a new stability will cause smaller coils, which may lead to bended fibers (Figure 5(c)) and even nonwoven fibers. Similar results can be seen in the PMMA fibers fabricated at the applied voltage of 4.5, 6.0, and $7.0 \mathrm{kV}$, respectively, as shown in Figure 6.

3.3. Polymer Concentration. The concentration of polymer solution has a significant influence on the formation of electrospun fibers. At low concentrations, solution surface tension has the dominant influence on fiber morphology and below a certain concentration drops or beads will form instead of fibers. At high concentrations, electrospinning will be prohibited by an inability to control and maintain the flow of a polymer solution to the nozzle [32]. So, in order to obtain electrospun fibers, the polymer solution should have an appropriate concentration. Figure 7 shows the optical microscope images of the electrospun PMMA fibers with various concentrations of 8.0,10.0,13.0, and $15.0 \mathrm{wt} \%$, respectively. Some disordered fibers with beads (Figure 7(a)) were observed at the low-concentration solution $(8.0 \mathrm{wt} \%)$. With the polymer concentration increasing to $13.0 \mathrm{wt} \%$, aligned PMMA fibers with uniform morphology were obtained, and, particularly, the alignment degree of fibers was subsequently increased (Figure $7(\mathrm{c})$ ) because the bending instability of the polymer jet could be suppressed by increasing the polymer concentration, and the jet travels in an approximate straight path toward the collector [20]. This makes it possible to collect highly aligned fibers on the collector. When the polymer concentration was increased to $15.0 \mathrm{wt} \%$, the PMMA fibers still displayed a good alignment.

As mentioned above, polymer solution with low concentration or low molecular weight could lead to the formation of microbeads or beaded fibers $[33,34]$. In this work, both PMMA and PVP nanofibers with beaded structures have been observed when spinning low-concentration PMMA/ THF (8.0 wt\%) and PVP/ethanol (8.0 wt\%) solutions, as shown in Figures 7(a) and 8(a). The PVP beaded fibers still kept a certain degree of orientation (Figure 8(a)). In order to observe the morphology of the beaded fibers clearly, we doped fluorescent rhodamine B into the PS solution (the concentration of PS/THF solution was $15.0 \mathrm{wt} \%$ ) for spinning. Figures 8(b)-8(c) show the SEM and fluorescent microscope images of rhodamine B/PS (1:50) beaded fibers electrospun at conditions of $5.0 \mathrm{kV}, 420 \mathrm{rpm}$, and $2.0 \mathrm{~cm}$. We can see that the micro-beads were aligned and uniformly dispersed in the fibers. However, when the concentration of PS/THF solution increased from 15.0 to $18.0 \mathrm{wt} \%$, micro-beads disappeared; only uniform and smooth fibers were observed, as shown in Figures $8(\mathrm{~d})-8(\mathrm{e})$. These results indicate that the morphology and structure of electrospun fibers can be also controlled by the polymer concentration. 


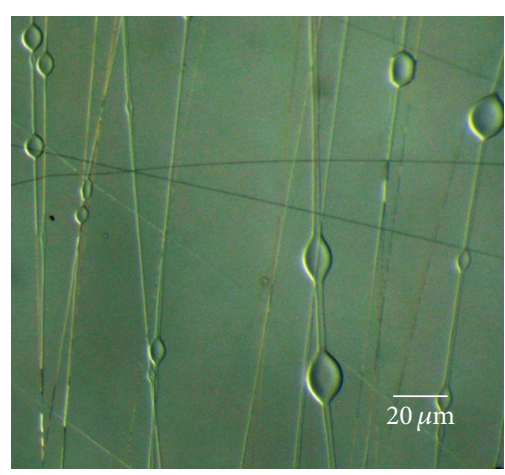

(a)

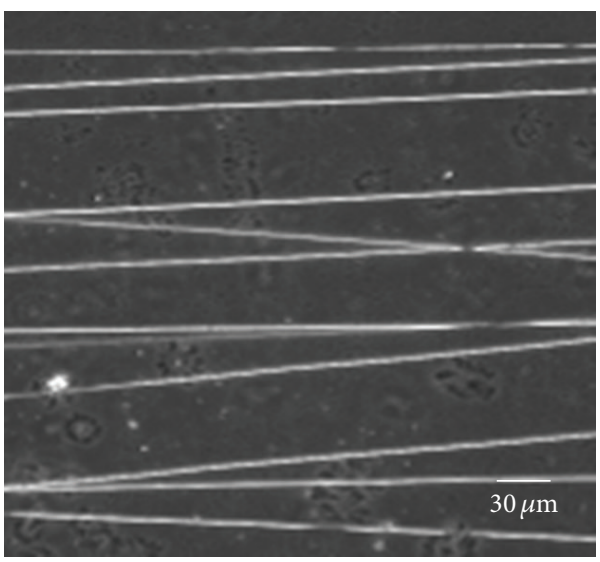

(d)

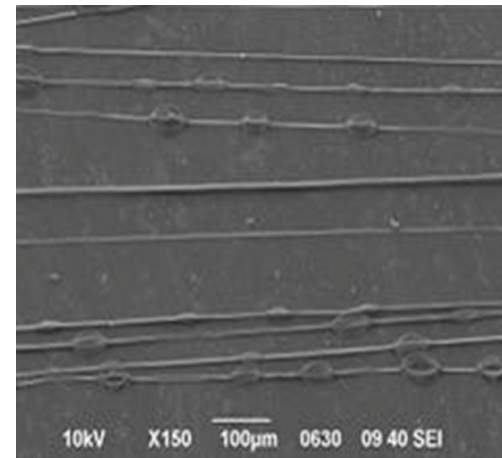

(b)

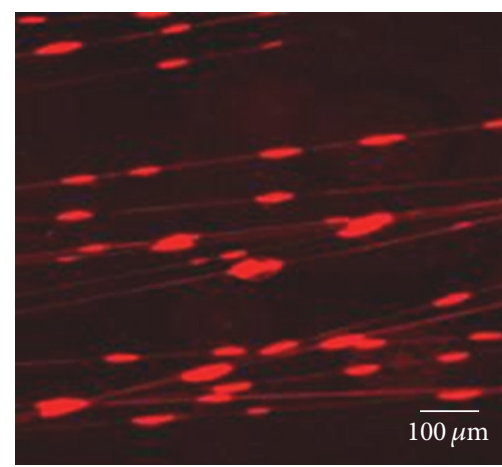

(c)

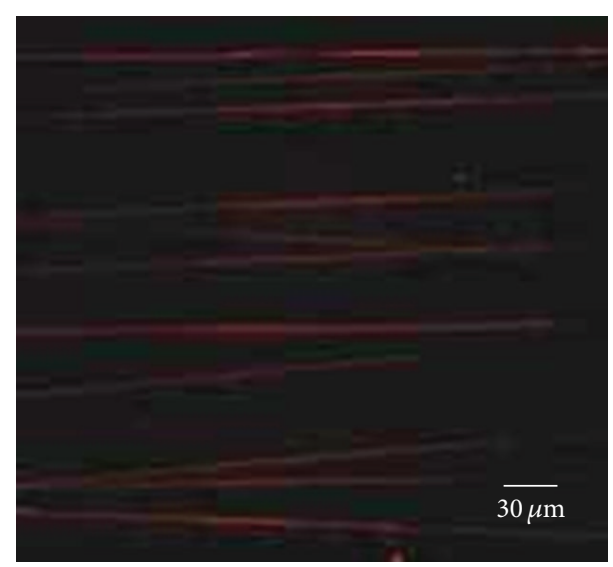

(e)

Figure 8: (a) Optical microscope image of PVP beaded fibers electrospun at conditions of $2.8 \mathrm{kV}, 420 \mathrm{rpm}$, and $3.0 \mathrm{~cm}$ (the concentration of PVP/ethanol was $8.0 \mathrm{wt} \%$ ). (b) SEM and (c) fluorescent microscope images of rhodamine B/PS beaded fibers electrospun at conditions of $5.0 \mathrm{kV}, 420 \mathrm{rpm}$, and $2.0 \mathrm{~cm}$ (the concentration of PS/THF solution was $15.0 \mathrm{wt} \%$ ). (d) Optical and (e) fluorescent microscope images of rhodamin B/PS uniform microfibers (the concentration of PS/THF solution was $18.0 \mathrm{wt} \%$ ).

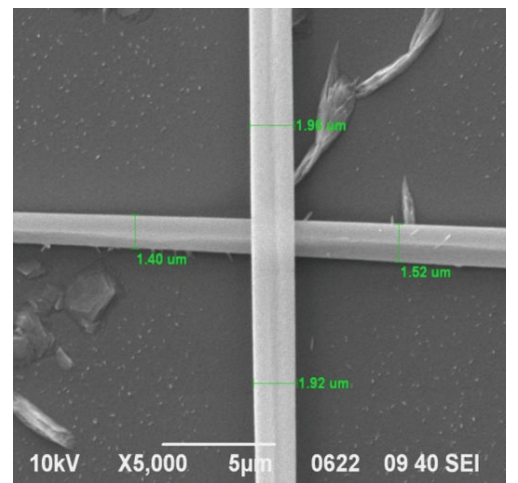

(a)

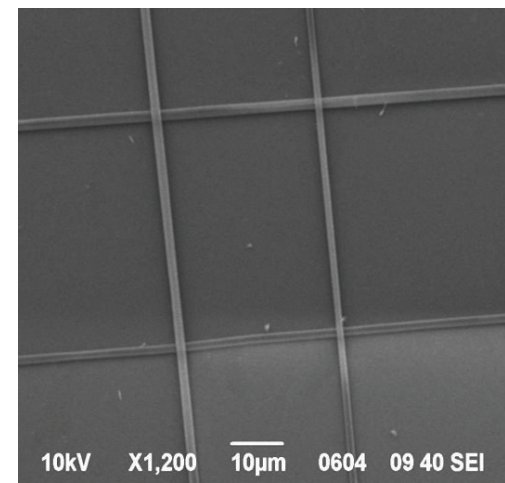

(b)

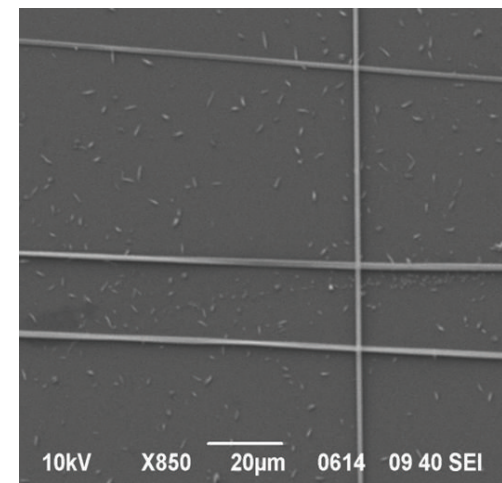

(c)

FIGURE 9: SEM images of cross-aligned PS fiber arrays with angle of $90^{\circ}$ fabricated by centrifugal electrospinning: (a) $1 \times 1$, (b) $2 \times 2$, and (c) $1 \times 3$.

3.4. Assembly of Crossed Polymer Fibers. Cross-aligned semiconducting fibers have potential applications in electronic and optoelectronic devices such as logic gates, computational circuits [35-37], multicolor light-emitting diodes [36, 38], and solar cells $[39,40]$. In this work, besides highly aligned fibers in a large area, crossed polymer fiber arrays have also been assembled by our new centrifugal electrospinning setup. Figure 9 shows the crossed two-layer nanofibers arrays with the angle of $90^{\circ}$ collected by a two-step spinning (rotating substrate after the collection of the first layer and then spinning again). The crossed angle is adjustable according to the rotation of glass substrate with the first layer of aligned 


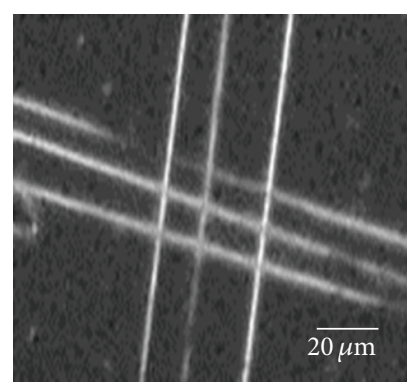

(a)

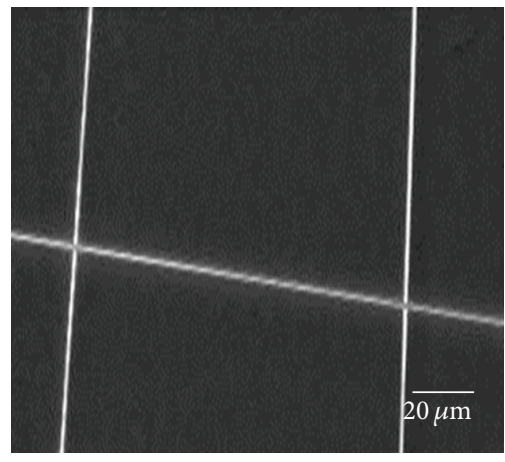

(e)

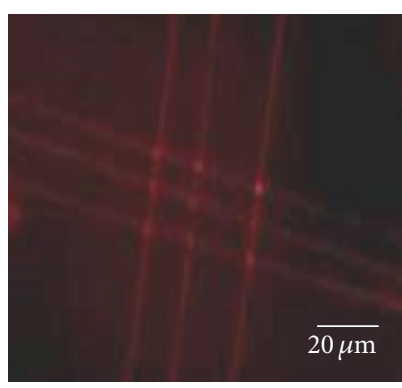

(b)

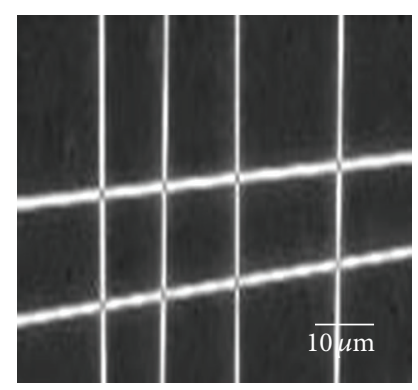

(c)

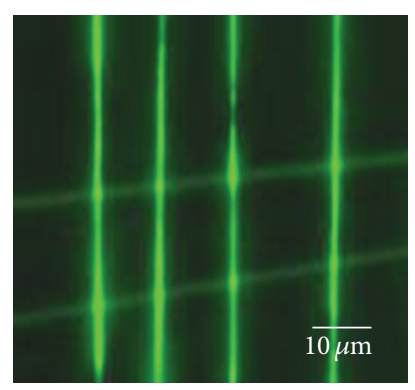

(d)

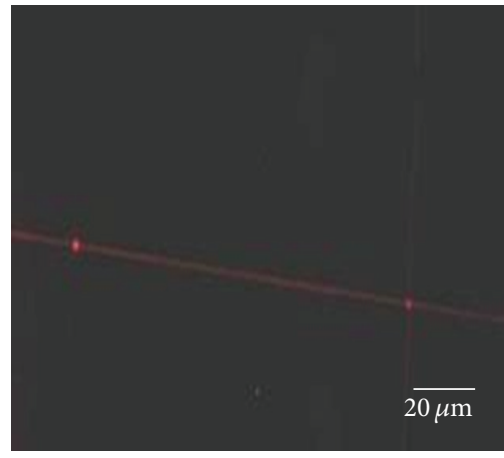

(f)

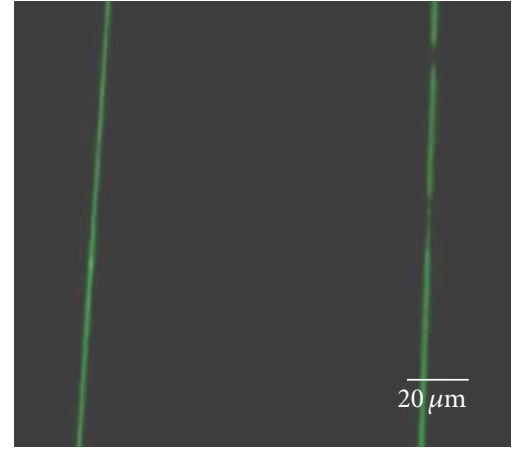

(g)

FIGURE 10: ((a) and (c)) Optical and ((b) and (d)) fluorescent microscope images of rhodamin B/PS (b) and fluorescein/PS (d) crossaligned fiber arrays. (e) Optical and ((f) and (g)) fluorescent microscope images of a two-layer fiber array composed of rhodamin B/PS and fluorescein/PS fibers.

fibers. When the axis of aligned fibers is perpendicular to the moving direction of the needle, crossed nanofibers with the angle of $90^{\circ}$ are achieved. Furthermore, fluorescent fiber arrays with crossed structure were assembled when we doped different fluorescent materials (e.g., rhodamin B (Figures 10(a)-10(b)) and fluorescein (Figures 10(c)-10(d))) into the spun solutions. Their optical and fluorescent microscope images are clearly shown in Figures 10(a)-10(d), respectively. Particularly, a two-layer array of PS fibers doped with rhodamin B and fluorescein, separately, were also fabricated by the two-step spinning (Figures 10(e)-10(g)). This structure may have potential application in electrical, optical, or biological devices.

\section{Conclusions}

In summary, a novel centrifugal electrospinning technique has been proposed. This technique combines the advantages of centrifugal spinning and electrospinning and only requires a lower working voltage $(2.8-6.0 \mathrm{kV})$, a slower rotational speed (360-540 rpm), and a shorter spinning distance (2.0$4.0 \mathrm{~cm}$ ). Through optimizing the experimental parameters such as working voltage, rotational speed, collecting distance, and solution concentration, highly aligned arrays of ultrafine polymer fibers in a large area have been obtained. It is found that more than $97.7 \%$ of the polymer fibers are within $\theta=5^{\circ}$, and all the rest of them are still within $\theta=10^{\circ}$. Beside aligned straight fibers, cross-aligned arrays of ultrafine polymer fibers have also been successfully assembled. These results indicate that centrifugal electrospinning is an efficient method to assemble aligned ultrafine fibers which can be used in electronic and optoelectronic devices.

\section{Acknowledgments}

This work was financially supported by the National Natural Science Foundation of China (11074138, 11004114, and 51373082), Taishan Scholars Program of Shandong Province, China (ts20120528), Natural Science Foundation of Shandong Province for Distinguished Young Scholars (JQ201103), National Key Basic Research Development Program of China (973 special preliminary study plan, 2012CB722705), Project of Shandong Province Higher Educational Science and Technology Program (J13LJ07), Program of Science and Technology in Qingdao City (13-1-4-195-jch), and Program for Scientific Research Innovation Team in Colleges and Universities of Shandong Province.

\section{References}

[1] Y. Xia, P. Yang, Y. Sun et al., "One-dimensional nanostructures: synthesis, characterization, and applications," Advanced Materials, vol. 15, no. 5, pp. 353-389, 2003.

[2] X. Liu, Y.-Z. Long, L. Liao, X. Duan, and Z. Fan, "Large-scale integration of semiconductor nanowires for high-performance flexible electronics," ACS Nano, vol. 6, no. 3, pp. 1888-1900, 2012.

[3] Y.-Z. Long, M.-M. Li, C. Gu et al., "Recent advances in synthesis, physical properties and applications of conducting polymer 
nanotubes and nanofibers," Progress in Polymer Science, vol. 36, no. 10, pp. 1415-1442, 2011.

[4] M. Yu, Y.-Z. Long, B. Sun, and Z. Fan, "Recent advances in solar cells based on one-dimensional nanostructure arrays," Nanoscale, vol. 4, no. 9, pp. 2783-2796, 2012.

[5] Y. Z. Long, M. Yu, B. Sun, C. Z. Gu, and Z. Y. Fan, "Recent advances in large-scale assembly of semiconducting inorganic nanowires and nanofibers for electronics, sensors and photovoltaics," Chemical Society Reviews, vol. 41, no. 12, pp. 45604580, 2012.

[6] Z.-M. Huang, Y.-Z. Zhang, M. Kotaki, and S. Ramakrishna, "A review on polymer nanofibers by electrospinning and their applications in nanocomposites," Composites Science and Technology, vol. 63, no. 15, pp. 2223-2253, 2003.

[7] D. Li, Y. Wang, and Y. Xia, "Electrospinning of polymeric and ceramic nanofibers as uniaxially aligned arrays," Nano Letters, vol. 3, no. 8, pp. 1167-1171, 2003.

[8] B. S. Jha, R. J. Colello, J. R. Bowman et al., “Two pole air gap electrospinning: fabrication of highly aligned, three-dimensional scaffolds for nerve reconstruction," Acta Biomaterialia, vol. 7, no. 1, pp. 203-215, 2011.

[9] J.-S. Tan, Y.-Z. Long, and M.-M. Li, "Preparation of aligned polymer micro/nanofibres by electrospinning," Chinese Physics Letters, vol. 25, no. 8, pp. 3067-3070, 2008.

[10] B. Sundaray, V. Subramanian, T. S. Natarajan, R.-Z. Xiang, C.-C. Chang, and W.-S. Fann, "Electrospinning of continuous aligned polymer fibers," Applied Physics Letters, vol. 84, no. 7, pp. 12221224, 2004.

[11] M. K. Shin, Y. J. Kim, S. I. Kim et al., "Enhanced conductivity of aligned PANi/PEO/MWNT nanofibers by electrospinning," Sensors and Actuators B, vol. 134, no. 1, pp. 122-126, 2008.

[12] Y. Z. Long, J. Zheng, F. Shao, W. H. Han, H. X. Yin, and Z. $\mathrm{H}$. Zhang, "An electrospinning setup with a modified doubleframe collector for fabrication of helical twisted nanofibers," China Patent ZL201110137420.0, 2011.

[13] J. Rafique, J. Yu, J. L. Yu et al., "Electrospinning highly aligned long polymer nanofibers on large scale by using a tip collector," Applied Physics Letters, vol. 91, no. 6, Article ID 063126, 2007.

[14] G. H. Kim, "Electrospun PCL nanofibers with anisotropic mechanical properties as a biomedical scaffold," Biomedical Materials, vol. 3, no. 2, pp. 25010-25018, 2008.

[15] M. Acharya, G. K. Arumugam, and P. A. Heiden, "Dual electric field induced alignment of electrospun nanofibers," Macromolecular Materials and Engineering, vol. 293, no. 8, pp. 666674, 2008.

[16] D. Yang, J. Zhang, and J. Nie, "Aligned electrospun nanofibers induced by magnetic field," Journal of Applied Polymer Science, vol. 110, no. 6, pp. 3368-3372, 2008.

[17] H. Wang, H. Tang, J. He, and Q. Wang, "Fabrication of aligned ferrite nanofibers by magnetic-field-assisted electrospinning coupled with oxygen plasma treatment," Materials Research Bulletin, vol. 44, no. 8, pp. 1676-1680, 2009.

[18] F. Dabirian, S. Sarkeshik, and A. Kianiha, "Production of uniaxially aligned nanofibers using a modified electrospinning method: rotating jet," Current Nanoscience, vol. 5, no. 3, pp. 318323, 2009.

[19] C.-C. Liao, S.-S. Hou, C.-C. Wang, and C.-Y. Chen, "Electrospinning fabrication of partially crystalline bisphenol A polycarbonate nanofibers: the effects of molecular motion and conformation in solutions," Polymer, vol. 51, no. 13, pp. 28872896, 2010.
[20] F. Dabirian, S. A. Hosseini Ravandi, A. R. Pishevar, and R. A. Abuzade, "A comparative study of jet formation and nanofiber alignment in electrospinning and electrocentrifugal spinning systems," Journal of Electrostatics, vol. 69, no. 6, pp. 540-546, 2011.

[21] M. Khamforoush and M. Mahjob, "Modification of the rotating jet method to generate highly aligned electrospun nanofibers," Materials Letters, vol. 65, no. 3, pp. 453-455, 2011.

[22] M. Li, Y.-Z. Long, D. Yang et al., "Fabrication of one dimensional superfine polymer fibers by double-spinning," Journal of Materials Chemistry, vol. 21, no. 35, pp. 13159-13162, 2011.

[23] D. Zhang and J. Chang, "Patterning of electrospun fibers using electroconductive templates," Advanced Materials, vol. 19, no. 21, pp. 3662-3667, 2007.

[24] R. T. Weitz, L. Harnau, S. Rauschenbach, M. Burghard, and K. Kern, "Polymer nanofibers via nozzle-free centrifugal spinning," Nano Letters, vol. 8, no. 4, pp. 1187-1191, 2008.

[25] M. R. Badrossamay, H. A. McIlwee, J. A. Goss, and K. K. Parker, "Nanofiber assembly by rotary jet-spinning," Nano Letters, vol. 10, no. 6, pp. 2257-2261, 2010.

[26] T. Senthilram, L. A. Mary, J. R. Venugopal, L. Nagarajan, S. Ramakrishna, and V. R. G. Dev, "Self crimped and aligned fibers," Materials Today, vol. 14, no. 5, pp. 226-229, 2011.

[27] H.-X. Yin, Y.-Z. Long, F. Yu, S.-J. Zhao, and X.-L. She, "Electrospun europium complex/polymer composite microfibers and its modified photoluminescence properties," Materials Science Forum, vol. 688, pp. 74-79, 2011.

[28] D. H. Reneker and A. L. Yarin, "Electrospinning jets and polymer nanofibers," Polymer, vol. 49, no. 10, pp. 2387-2425, 2008.

[29] T. Han, D. H. Reneker, and A. L. Yarin, "Buckling of jets in electrospinning," Polymer, vol. 48, no. 20, pp. 6064-6076, 2007.

[30] S. A. Theron, E. Zussman, and A. L. Yarin, "Experimental investigation of the governing parameters in the electrospinning of polymer solutions," Polymer, vol. 45, no. 6, pp. 2017-2030, 2004.

[31] T. D. Brown, P. D. Dalton, and D. W. Hutmacher, "Direct writing by way of melt electrospinning," Advanced Materials, vol. 23, no. 47, pp. 5651-5657, 2011.

[32] J. M. Deitzel, J. Kleinmeyer, D. Harris, and N. C. Beck Tan, “The effect of processing variables on the morphology of electrospun nanofibers and textiles," Polymer, vol. 42, no. 1, pp. 261-272, 2001.

[33] R. Lalani and L. Liu, "Synthesis, characterization, and electrospinning of zwitterionic poly(sulfobetaine methacrylate)," Polymer, vol. 52, no. 23, pp. 5344-5354, 2011.

[34] J. Zheng, Y.-Z. Long, B. Sun et al., "Polymer nanofibers prepared by low-voltage near-field electrospinning," Chinese Physics $B$, vol. 21, no. 4, Article ID 048102, 2012.

[35] H. Yan, H. S. Choe, S. Nam et al., "Programmable nanowire circuits for nanoprocessors," Nature, vol. 470, no. 7333, pp. 240244, 2011.

[36] Y. Huang and C. M. Lieber, "Integrated nanoscale electronics and optoelectronics: exploring nanoscale science and technology through semiconductor nanowires," Pure and Applied Chemistry, vol. 76, no. 12, pp. 2051-2068, 2004.

[37] Q. Tang, Y. Tong, W. Hu, Q. Wan, and T. Bjørnholm, "Assembly of nanoscale organic single-crystal cross-wire circuits," Advanced Materials, vol. 21, no. 42, pp. 4234-4237, 2009.

[38] Y. Huang, X. Duan, and C. M. Lieber, "Nanowires for integrated multicolor nanophotonics," Small, vol. 1, no. 1, pp. 142-147, 2005. 
[39] H.-S. Shim, S.-I. Na, S. H. Nam et al., "Efficient photovoltaic device fashioned of highly aligned multilayers of electrospun $\mathrm{TiO}_{2}$ nanowire array with conjugated polymer," Applied Physics Letters, vol. 92, no. 18, Article ID 183107, 2008.

[40] S. Wu, Q. Tai, and F. Yan, "Hybrid photovoltaic devices based on poly (3-hexylthiophene) and ordered electrospun $\mathrm{ZnO}$ nanofibers," Journal of Physical Chemistry C, vol. 114, no. 13, pp. 6197-6200, 2010. 

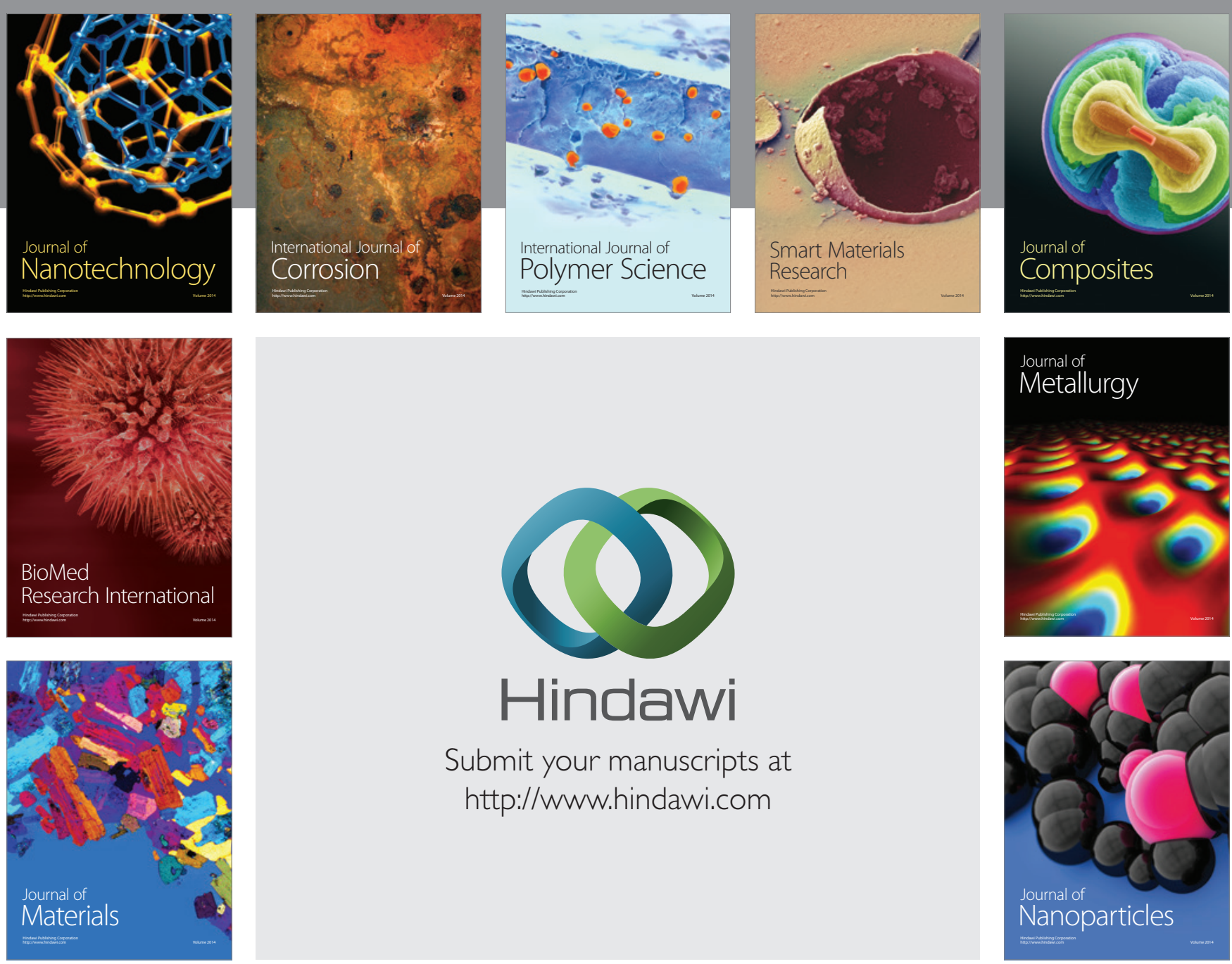

Submit your manuscripts at http://www.hindawi.com
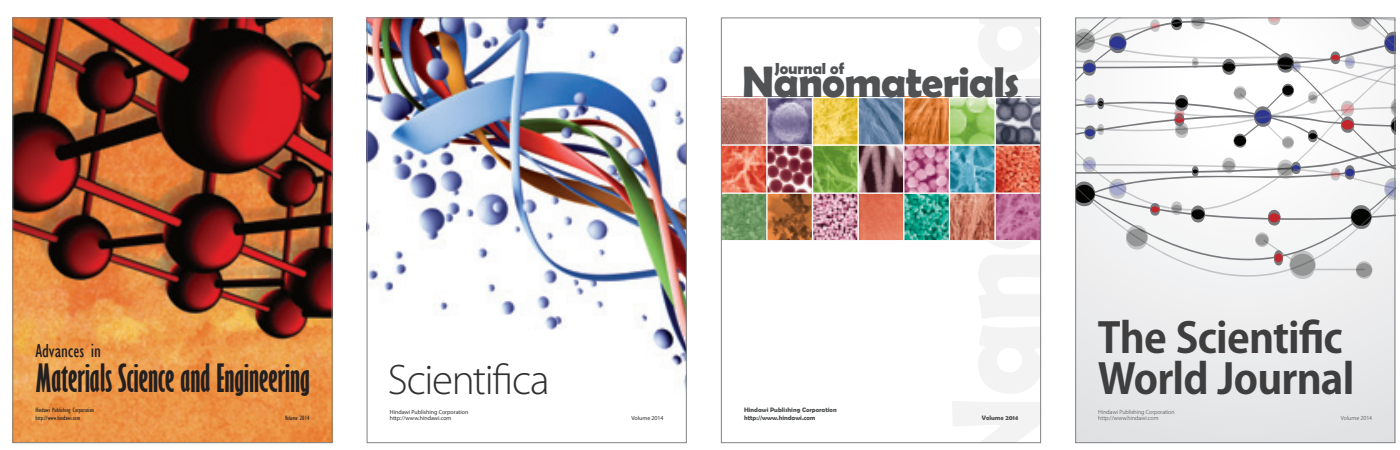

\section{The Scientific World Journal}
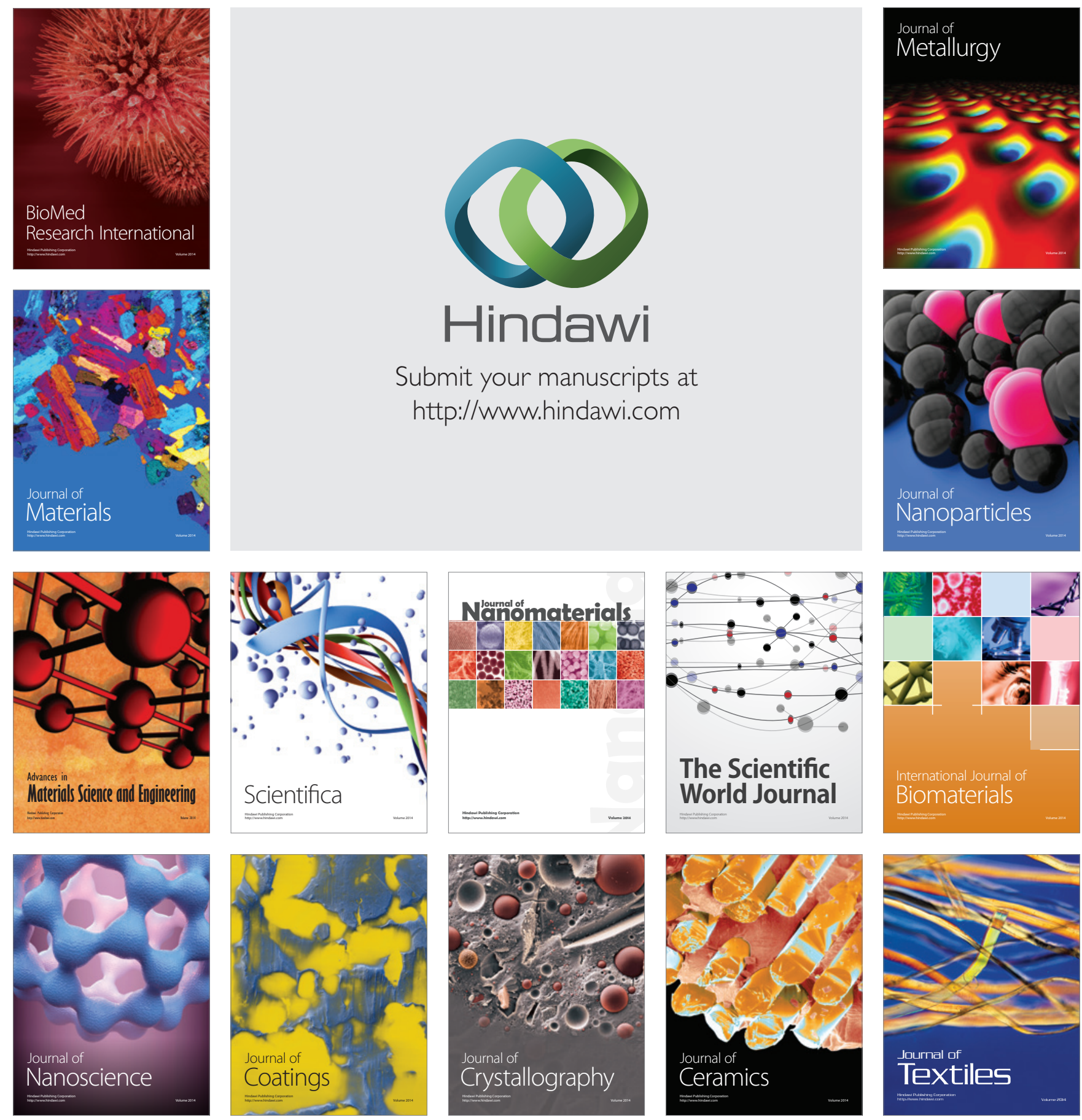